\title{
Field-enabled quantum interference in atomic Auger decay
}

\author{
Murali Krishna Ganesa Subramanian $\odot,{ }^{1,2}$ Roman Brannath, ${ }^{2}$ Ralph Welsch $\odot,{ }^{1}$ Robin Santra $\odot,{ }^{1,2}$ and Markus Drescher $\circledast^{2}$ \\ ${ }^{1}$ Center for Free-Electron Laser Science, Deutsches Elektronen-Synchrotron DESY, 22607 Hamburg, Germany \\ ${ }^{2}$ Department of Physics, Universität Hamburg, 22761 Hamburg, Germany
}

(Received 9 June 2020; accepted 17 July 2020; published 11 August 2020)

\begin{abstract}
We demonstrate that an external terahertz $(\mathrm{THz})$ field enables the formation of interference between two distinct Auger pathways leading to the same final ionic state. The kinetic energy of Auger electrons ejected from either of two spin-orbit split one-hole states of magnesium cations is recorded. In the presence of the THz field, a clear oscillatory structure in the Auger spectrum emerges, which we find to be in very good agreement with an analytical model based on perturbation theory. For this interference to occur, the THz field has to chirp the energy of both Auger electrons and photoelectrons simultaneously, in order to create states with indistinguishable quantum properties.
\end{abstract}

DOI: 10.1103/PhysRevA.102.022807

\section{INTRODUCTION}

X-ray-induced innershell processes like photoionization of core electrons, Auger decay, or x-ray fluorescence are widespread tools to probe structural changes in atoms, molecules, and solids with element specificity [1,2]. Advances in X-ray light sources such as X-ray free-electron lasers and table-top sources using high harmonic generation make it possible to tackle problems at the forefront of science using ultrafast x-ray techniques that can achieve atomic spatial resolution and femtosecond time resolution [3]. In this context, external fields have been used to modify or control $\mathrm{x}$-ray-induced innershell processes to obtain a more detailed picture of the investigated processes and to allow for new applications. For example, x-ray diffraction can be optically modulated allowing for the investigation of optically induced charges [4], innershell photoabsorption can be controlled by nonperturbative modification of the level structure of the bound electrons $[5,6]$ or by laser-induced molecular alignment $[7,8]$, line profiles of photoabsorption can be modified $[9,10]$, and the streaking of ejected photoelectrons with near- or far-infrared light or the observation of circular dichroism is a useful tool in characterizing $\mathrm{x}$-ray pulses [11-13].

A particularly interesting probe of the electronic structure of matter is based on the Auger effect, i.e., the nonradiative decay of a deeply bound core hole through the emission of an Auger electron. The recorded kinetic energy of the Auger electrons is an intrinsic property of the electronic structure of the target and does not depend on the energy of the incoming light. Therefore, Auger spectroscopy is an important and widely used probe of the electronic structure of atoms, molecules, and surfaces. The emission of Auger electrons and photoelectrons can be further probed or controlled by intense laser fields, e.g., in the infrared (IR) energy range. In such laser-assisted processes, the system is irradiated by an extreme-ultraviolet (EUV) or x-ray pulse, which creates a core hole and a photoelectron. The core hole can decay nonradiatively and an Auger electron is emitted. The emitted electrons are subsequently dressed by the applied intense laser field.
This leads, e.g., to the appearance of several side bands or to a continuous shift of the spectrum. Laser-assisted photoelectric effect [14-19], laser-assisted Auger decay [20,21], and laserenabled Auger decay [22-24] have been observed. Recent technological advances allow for the creation of intense laser fields in the far-IR [or terahertz (THz)] energy range, which can be well synchronized with EUV or X-ray pulses [25,26]. This enables the study of laser-assisted Auger decay also in the $\mathrm{THz}$ regime [27].

Notably, if the final state can be reached via different quantum pathways, interference may occur. Various mechanisms underlying such interference in atomic or molecular systems have been identified. Upon scanning the energy of the photoelectron across the fixed energy of the Auger electron, both electrons can be made indistinguishable, thus creating interference in their spectra and angular distributions [28]. Also different resonant Auger transitions may accidentally have significant spectral overlap, thus giving rise to interference [29,30]. Spectral separation between Auger electrons may be partially bridged by postcollision interaction, predicted already in 1977 [31], but experimentally verified only in 2001 [32]. Molecular targets introduce additional channels, e.g., via lifetime-vibrational interference [33,34], where energetic degeneracy of electronic states is reached through nuclear motion.

All of these cases rely on a specific intrinsic electronic structure, whereas in the present work we demonstrate how interference is enabled through external control. Under conditions found in atomic magnesium used as an example, a timevarying light field can chirp the energy of Auger electrons and photoelectrons emitted from a spin-orbit split state so as to induce interference between two distinct Auger pathways that natively form two separate spectral peaks.

\section{EXPERIMENT}

In the experiment, an initial $2 p$ core hole is formed in $\mathrm{Mg}$ atoms by photoionization using ultrashort EUV pulses at $91 \mathrm{eV}$ of photon energy, generated as high harmonics of 


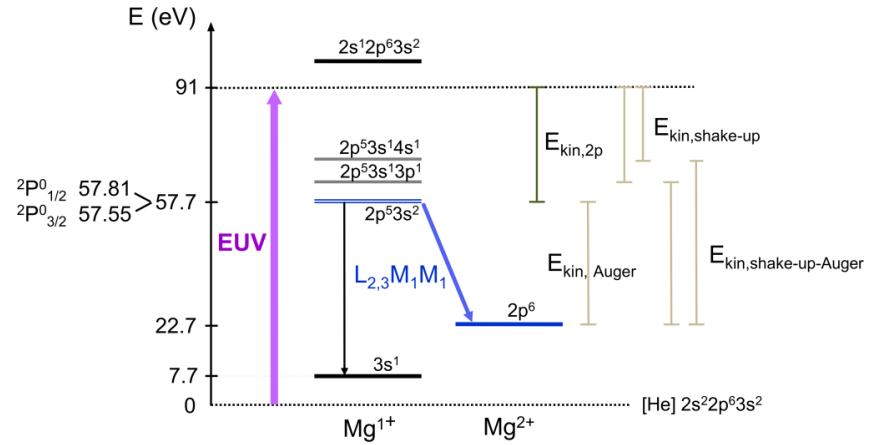

FIG. 1. Energy level diagram of Mg and its cations. Nonresonant excitation leads to core-excited states that relax upon emission of LMM Auger electrons. The excess kinetic energy of emitted photoelectrons and Auger electrons is indicated by vertical bars.

800-nm, 38-fs, 2-mJ pulses from a 1-kHz Ti:sapphire laser system in a neon-gas target after compression of the fundamental pulses in a neon-filled hollow fiber [35]. Near-infrared pulses of $1 \mathrm{~mJ}$ of energy from the same laser are used to generate $\mathrm{THz}$ radiation by optical rectification in a $\mathrm{LiNbO}_{3}$ crystal. Phase-matching between near- and far-infrared light fields by pulse-front tilting [36] yields $0.5-\mu \mathrm{J}$ single-cycle $\mathrm{THz}$ pulses centered at $0.6 \mathrm{THz}$. After temporal synchronization with a tunable optical delay line, EUV and THz pulses are focused into an effusive beam of $\mathrm{Mg}$ atoms formed a few millimeters above the orifice of a metal oven operated at up to $700{ }^{\circ} \mathrm{C}$. The ejected photoelectrons and Auger electrons are collected in an approximately $20^{\circ}$ solid angle perpendicular to the light propagation and guided into a time-of-flight spectrometer. Owing to an alignment of the spectrometer axis with the polarization of the $\mathrm{THz}$ beam, the escaping electrons are energetically streaked according to the phase of the $\mathrm{THz}$ vector potential at the instant of emission [25].

Figure 1 depicts the relevant energy levels of atomic magnesium. At $91 \mathrm{eV}$ of photon energy, a $2 p$ core electron is promoted into the ionization continuum. The $2 p^{5} 3 s^{2}$ level of $\mathrm{Mg}^{+}$is spin-orbit split by $0.28 \mathrm{eV}$ and thus the decay of the intermediate hole states creates Auger electrons at 34.87 and $35.15 \mathrm{eV}$, respectively. The corresponding Auger lifetimes deduced from the literature vary considerably between a lower bound of about $20 \mathrm{fs}$, resulting from spectral linewidth measurements [37,38], and 500 fs predicted theoretically [31]. Regardless of their actual resolution in the experiment, natively emitted Auger electrons from $\mathrm{Mg}^{+}$ions form distinct peaks in the recorded kinetic energy spectrum and will thus not be able to interfere.

Figure 2 presents the unperturbed and the THz-streaked kinetic energy spectra of the ejected electrons. The unperturbed spectrum clearly shows the photoelectron peak around $32.5 \mathrm{eV}$ and the Auger peak around $35 \mathrm{eV}$. Please note that with the given spectral resolution of the utilized time-of-flight spectrometer of $>0.3 \mathrm{eV}$ the fine structure of the $L M M$ Auger feature, i.e., the two distinct Auger pathways under consideration, cannot be resolved in the unperturbed spectrum. The width of the $2 p$ photoline is governed by the bandwidth of the exciting radiation, dictated by the $2-\mathrm{eV}$ bandpass of the EUV multilayer mirror used for harmonic selection. The

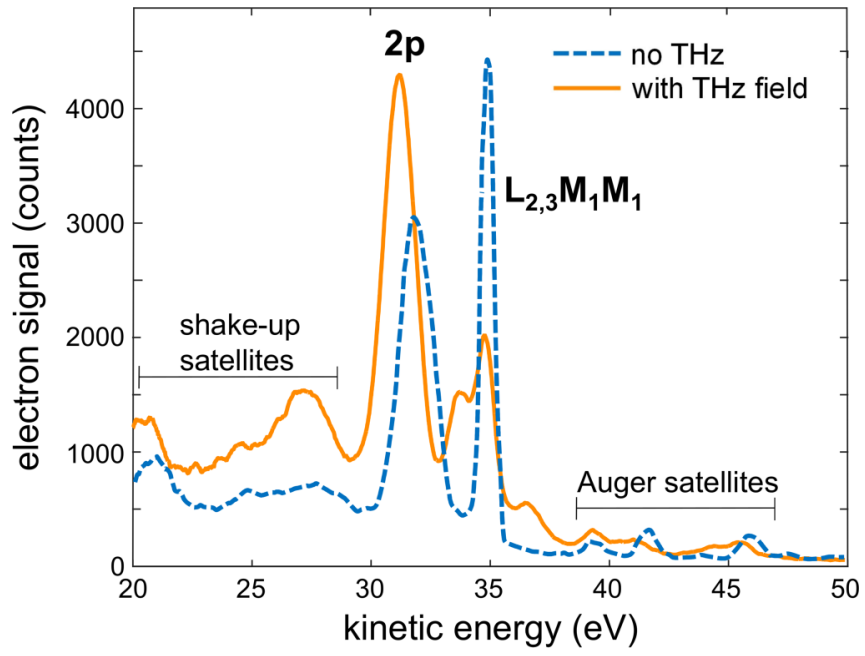

FIG. 2. Electron kinetic energy spectrum of atomic $\mathrm{Mg}$ after excitation at $91 \mathrm{eV}$ of photon energy without (dashed blue line) and with (solid orange line) applied THz field.

application of the $\mathrm{THz}$ field significantly alters the Auger spectrum when the relative timing with respect to the EUV pulse corresponds to a steep slope of the $\mathrm{THz}$ vector potential while the electron wave packet is formed in the continuum. Rather than a plain broadening expected for regular streaking, the spectrum displays a clear oscillatory structure reminiscent of an interference pattern.

\section{THEORY}

To investigate whether and in what form the oscillatory structure in the spectrum results from quantum interference, we employ an analytical model based on time-dependent perturbation theory. In the present approach, the interaction of Mg atoms with the EUV pulse as well as the Auger decay are treated perturbatively. The strong $\mathrm{THz}$ field, however, is treated in a nonperturbative fashion. Within this model (for details of the derivation, see Appendices A and B) the probability of finding the Auger electron in state $\alpha$ is given as

$$
\begin{aligned}
P_{\alpha}= & \lim _{t \rightarrow \infty} \sum_{\substack{\gamma>\beta \\
\Pi(\Pi<\alpha)}} \mid \sum_{a^{\prime \prime}} \sum_{j} \int_{-\infty}^{t} d t^{\prime} \\
& \times \int_{-\infty}^{t^{\prime}} d t^{\prime \prime} u_{\alpha a^{\prime \prime}}\left(t, t^{\prime}\right) e^{-i I_{\beta \gamma}\left(t-t^{\prime}\right)} \\
& \times\left. v_{a^{\prime \prime} j \beta \gamma} u_{\Pi a}\left(t, t^{\prime \prime}\right) e^{-i\left(I_{j}-i \frac{\Gamma_{j}}{2}\right)\left(t^{\prime}-t^{\prime \prime}\right)} \varepsilon_{\mathrm{EUV}}\left(t^{\prime \prime}\right) z_{a j}\right|^{2},
\end{aligned}
$$

where the $t^{\prime \prime}$ integral corresponds to photoionization and the $t^{\prime}$ integral corresponds to Auger decay, with $t^{\prime}>t^{\prime \prime}$. The onebody matrix element $z_{a j}$ determines the creation of a hole at time $t^{\prime \prime}$, in which $j$ corresponds to the state of the hole and $a$ corresponds to the state of the emitted photoelectron, with ionization energy $I_{j}=-\epsilon_{j}$. The photoionizing EUV pulse has 


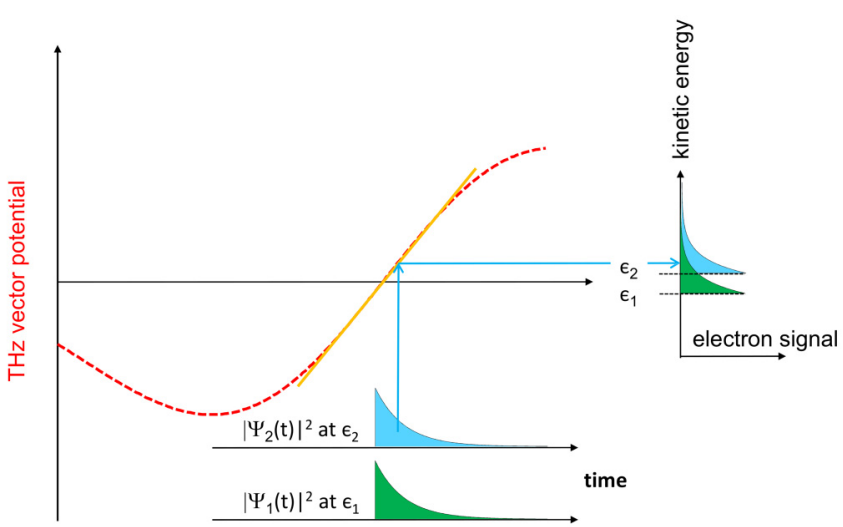

FIG. 3. Principle of field-enabled interference of Auger pathways of different initial Auger energies $\epsilon_{1}$ and $\epsilon_{2}$. The vector potential of the THz field (dashed curve) imprints the same gradual change (almost linear chirp) of the instantaneous frequency onto both wave packets, thus creating partial energetic degeneracy that may lead to interference, if they carry a defined phase relationship.

an electric field of $\varepsilon_{\mathrm{EUV}}\left(t^{\prime \prime}\right)$. The term $\Gamma_{j}=1 / \tau_{j}$ corresponds to the decay rate of the hole in state $j$. The emitted photoelectron evolves from state $a$ at time $t^{\prime \prime}$ to state $\Pi$ at time $t$ under the influence of the THz field, which is described by the term $u_{\Pi a}\left(t, t^{\prime \prime}\right)$. The two-body matrix element $v_{a^{\prime \prime} j \beta \gamma}$ determines the Auger-decay step. The initially created state with a hole in $j$ decays to a state with a hole in $\gamma$ and a simultaneously emitted Auger electron emitted from $\beta$ into state $a^{\prime \prime}$. Now, the emitted Auger electron evolves under the influence of the THz field from state $a^{\prime \prime}$ at time $t^{\prime}$ to state $\alpha$ at final time $t$, which is captured by $u_{\alpha a^{\prime \prime}}\left(t, t^{\prime}\right)$. The different dicationic states described by the two holes in $\beta$ and $\gamma$ and the state of the photoelectron $\Pi$ contribute incoherently to the signal. Please note that a full $a b$ initio simulation of the process is not feasible as, e.g., both the photoelectron and the Auger electron are unbound and prohibitively large spatial grids are required to simulate the unbound motion of two electrons ejected from an atom.

To observe interference between the two Auger pathways discussed above, the whole system has to evolve into the same indistinguishable final states, i.e., the combined quantum states of the Auger electron, the photoelectron, and the dication have to be indistinguishable. For the dication, this is fulfilled here as both Auger pathways lead to the same final state. Therefore, to observe interference, the applied $\mathrm{THz}$ field has to modify both the photoelectron and the Auger electron. Since the large bandwidth of the EUV radiation blurs any substructure in the $2 p$ photoelectron peaks, we focus the detailed discussion of the streaking process on the Auger electrons. First, the $\mathrm{THz}$ pulse has to streak the energy of the emitted Auger electron from the $j_{1}=3 / 2$ and $j_{2}=1 / 2$ photoionization channels to overlap their energies. This can be understood by describing the emitted Auger electron in terms of partial waves with energies $\epsilon_{1}$ and $\epsilon_{2}$, but with the same temporal profile, i.e., an exponentially decaying transition probability (see Fig. 3). The exposure of these electron partial waves to a time-varying $\mathrm{THz}$ field, which has a period greater than the Auger lifetime, results in time-dependent momentum transfer from the $\mathrm{THz}$ field to the Auger electron.
In the experimental setup used in this work, the detection of the emitted electrons is restricted to a small subset of emission angles. This detection scheme does not allow for the detection of the different angular momentum states of the emitted electrons. If, however, a similar experiment were performed with a detection of electrons at all emission angles, one would have to take into account the distinct angular momenta of the Auger electrons. The spin-orbit split one-hole states under consideration have a total angular momentum of $j=1 / 2$ and $j=3 / 2$, respectively. As the final state of $\mathrm{Mg}^{2+}$, which is the same for both pathways, has an angular momentum of $j=0$, and the total angular momentum has to be conserved, the emitted Auger electrons have an angular momentum of $j=1 / 2$ and $j=3 / 2$, respectively. Therefore, to observe interference in such a case, the emitted Auger electrons should be indistinguishable in terms of all quantum numbers, and therefore, the perturbing $\mathrm{THz}$ field has to chirp the energy of the Auger electrons as well as modify their angular momentum.

Making a short-pulse approximation, i.e., assuming the EUV pulse to be a $\delta$ pulse, and taking the effect of the $\mathrm{THz}$ field into account through a linear frequency chirp, i.e., a quadratic temporal phase $e^{-i b t^{\prime 2}}$, we can define the transition amplitudes (for details, see Appendix B)

$$
\begin{aligned}
& \Psi_{\alpha \beta \gamma \Pi}\left(t^{\prime} ; t_{\mathrm{EUV}}\right) \\
& \quad= \begin{cases}\sum_{j} A_{j \alpha \beta \gamma \Pi} e^{-i\left(I_{j}-i \frac{\Gamma_{j}}{2}-I_{\beta \gamma}\right) t^{\prime}} e^{-i b\left(t^{\prime}+t_{\mathrm{EUV}}\right)^{2},}, & t^{\prime} \geqslant 0, \\
0, & t^{\prime}<0,\end{cases}
\end{aligned}
$$

and rewrite the spectrum given in Eq. (1) as a Fourier transform of these transition amplitudes:

$$
P_{\alpha}=\sum_{\substack{\gamma>\beta \\ \Pi(\Pi<\alpha)}}\left|\int_{-\infty}^{\infty} d t^{\prime} \Psi_{\alpha \beta \gamma \Pi}\left(t^{\prime} ; t_{\mathrm{EUV}}\right) e^{i \varepsilon_{\alpha} t^{\prime}}\right|^{2} .
$$

The parameter $t_{\mathrm{EUV}}$ represents the time at which the EUV pulse is centered, relative to the temporal evolution of the THz field.

\section{ANALYSIS}

In order to fit the experimental Auger spectral shape, we exploit that there is only a single Auger channel in the present problem and that the complex expansion coefficients $A_{j \alpha \beta \gamma} \Pi$ cannot change much over the narrow range of photoelectron (П) and Auger-electron $(\alpha)$ states considered. Hence, we write the Auger spectrum in the simplified form

$$
P_{\alpha}=\text { const. }\left|\sum_{j} A_{j} e^{-i \phi_{j}} \int_{0}^{\infty} d t^{\prime} e^{-i\left(E_{j}-i \frac{\Gamma_{j}}{2}-\varepsilon_{\alpha}\right) t^{\prime}} e^{-i b\left(t^{\prime}+t_{\mathrm{EUV}}\right)^{2}}\right|^{2},
$$

where $E_{3 / 2}=\epsilon_{1}=57.55 \mathrm{eV}$ and $E_{1 / 2}=\epsilon_{2}=57.81 \mathrm{eV}$ (cf. Fig. 1). In what follows, the phase difference $\Delta=\phi_{3 / 2}-$ $\phi_{1 / 2}$, the electronic chirp parameter $b$, and the Auger lifetime $\tau$, which is assumed to be the same for both pathways ( $\tau=$ $\left.\tau_{3 / 2}=\tau_{1 / 2}\right)$, serve as fit parameters. The branching ratio, i.e., 


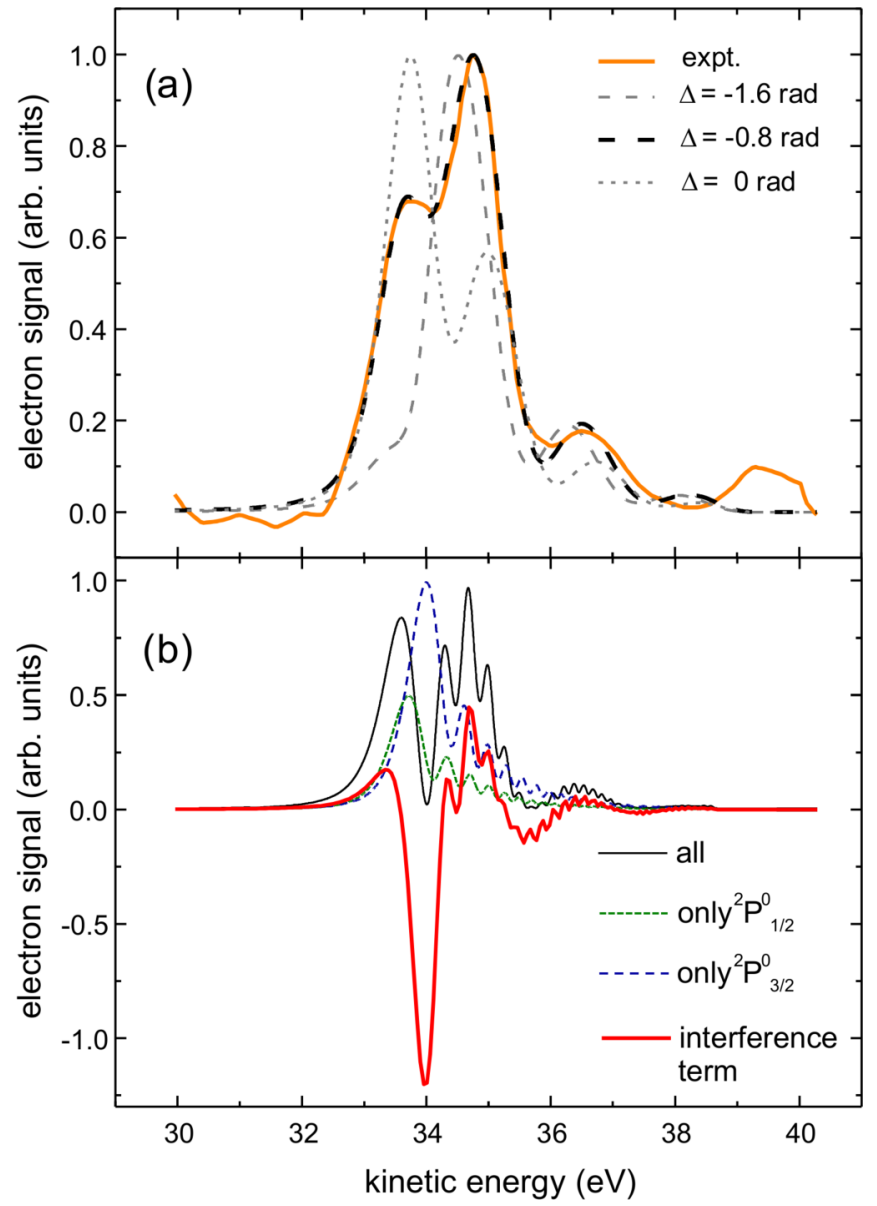

FIG. 4. (a) Experimental spectrum of streaked Mg LMM Auger lines (solid orange line) and simulation with the analytical model (dashed black line) with optimized parameters and with different values for the phase (gray, long dashed and short dashed lines). (b) The simulated spectrum is plotted for a $\delta$-pulse excitation (thin black), together with the two isolated Auger fine-structure components assuming no interference (long-dashed blue line and short-dashed green line). The interference term is shown as a thick red line.

$\left(A_{3 / 2} / A_{1 / 2}\right)^{2}$, is set to 2 , as given by the relative weight of the magnetic substates [37].

To obtain a meaningful comparison with experimental data, the spectrum obtained from Eq. (4) is convolved with a Gaussian shape capturing the broadening effects of a finite spectrometer resolution of $0.3 \mathrm{eV}$ and a finite duration of the EUV pulse. The resulting function is then fit to the experimental spectrum. Due to a residual detuning of the EUV-THz delay relative to the zero crossing of the $\mathrm{THz}$ vector potential, a small energy shift is added to the simulated spectrum.

The resulting best $t$, displayed in Fig. 4(a), is achieved for a phase difference $\Delta$ of $-0.8 \pm 0.6 \mathrm{rad}$, an Auger lifetime of $\tau=9.0(+5.2 /-3.8)$ fs, and an electronic chirp of $b=$ $119 \mathrm{meV} / \mathrm{fs}$, with the indicated errors resulting from a doubling of the residual between measured and simulated values. The combined spectral broadening of $0.6 \mathrm{eV}$ is compatible with an EUV pulse duration below 5 fs. The mutual relationship of the fitting parameters reveals a pronounced sensitivity of the observed spectral profile to the phase $\Delta$ between the electronic pathways of the two fine-structure components. This is exemplified in Fig. 4(a) by simulated spectra with the same set of parameters except phase differences set to $\Delta=0 \mathrm{rad}$ and $\Delta=-1.6 \mathrm{rad}$, respectively. The analytical model reproduces the experimental spectrum very well, which suggests that it captures the key features of the process.

In Fig. 4(b), the Gaussian convolution has been removed from the fitted spectrum $P_{\alpha}$ in order to further analyze the interference pattern. The full spectrum is decomposed into the two fine-structure components and the corresponding interference term. The sum over $j$ in Eq. (4) has two terms and we can thus expand it as

$$
P_{\alpha}=\left\{\left|z_{1}\right|^{2}+\left|z_{2}\right|^{2}+2 \mathcal{R}\left(z_{1} z_{2}^{*}\right)\right\} .
$$

The first two terms describe the two fine-structure components [blue and green lines in Fig. 4(b)], while the last term corresponds to the interference between the two Auger pathways [red line in Fig. 4(b)]. The total spectrum shows a clear, slowly oscillating interference pattern rather than two distinct Auger peaks. The superposition of the two electronic pathways results in a temporal beating at a time period $T=2 \pi /\left(\epsilon_{2}-\epsilon_{1}\right)$. Due to the streaking, this period is mapped to a difference in kinetic energy of $\delta E_{\text {kin }}=b \hbar T$. For the best-fit parameters this results in a kinetic energy difference of about $1.7 \mathrm{eV}$, which roughly matches the peak separation in Fig. 4. Moreover, the simulated spectrum now shows a noticeable, high-frequency oscillation pattern in the fine-structure components as well as in the interference term, which can be interpreted as fringes in the spectral domain resulting from the short-pulse approximation for the photoionization step. The rapid oscillations are suppressed if the spectrum is convolved as in Fig. 4(a).

\section{CONCLUSION}

In this paper, we have demonstrated that it is possible to create interference between two distinct Auger pathways by applying an intense $\mathrm{THz}$ field. Specifically, photoionization of $\mathrm{Mg}$ by an EUV pulse creates a hole in the $2 p$ shell. Thus, $\mathrm{Mg}^{+}$is prepared in either of two spin-orbit split one-hole states, which both decay to the same $\mathrm{Mg}^{2+}$ state by ejecting an Auger electron. When applying a THz field, we observed in the Auger spectrum interference between the associated electronic pathways. For this effect to manifest, not only the Auger-electron final states must be indistinguishable but also the same indistinguishability requirement applies to the photoelectron final states.

We interpreted the experimental spectrum by fitting an analytical model based on a perturbative expansion. By separating the model spectrum into its components, the importance of interference in explaining the experimentally observed spectral oscillations was clearly revealed. The analytical model agreed with the experimental data only if a significant nonvanishing phase difference between the interfering Auger pathways was taken into account. With the shape of the spectral peak being found to sensitively depend on the exact phase value, our method provides phase information from innershell processes that is difficult to obtain otherwise. We note, however, that this phase may contain contributions from the interaction of the outgoing electrons with the external $\mathrm{THz}$ field. Future work will focus on the isolation of intrinsic phases. 
Owing to the pronounced interference profile, the $\mathrm{Mg}^{+}$ Auger-decay lifetime could be extracted and was found to be at the lower bound of available predictions. In contrast to the examples of control given in the Introduction, the present approach shows how $\mathrm{THz}$ streaking may be employed to induce quantum interference in the electronic-decay pathways following multichannel innershell ionization.

\section{ACKNOWLEDGMENTS}

This work was financially supported by the Deutsche Forschungsgemeinschaft (DFG) within the framework of the SFB 925 "Light-Induced Dynamics and Control of Correlated Quantum Systems," Projects No. A1 and No. A5.

\section{APPENDIX A: THEORY AND DERIVATION}

The EUV photoionization and the Auger-decay process are treated within the framework of perturbation theory to arrive at a condition for interference of two Auger pathways in magnesium. The far-infrared (FIR) or terahertz (THz) field cannot be treated perturbatively because it interacts strongly with the Auger electrons and photoelectrons. In such a case the total Hamiltonian of the system is given by

$$
\hat{H}(t)=\hat{H}_{0}(t)+\lambda \hat{V}(t),
$$

where $\hat{H}_{0}(t)=\hat{H}_{0 \mathrm{~A}}-E_{0}+\hat{V}_{\mathrm{FIR}}(t)$ is the atomic Hamiltonian including the FIR field, with $\hat{H}_{0 \mathrm{~A}}$ being the unperturbed atomic Hamiltonian, $E_{0}$ the ground-state energy of the $\mathrm{Mg}$ atom, and $\hat{V}_{\mathrm{FIR}}(t)$ the FIR field. $\hat{V}(t)=\hat{V}_{\mathrm{EUV}}(t)+\hat{V}_{\mathrm{A}}$ is the perturbation which has contributions from EUV photoionization $\hat{V}_{\mathrm{EUV}}(t)$ and Auger decay $\hat{V}_{\mathrm{A}}$. Please note that we have assumed atomic units throughout the derivation, i.e., $\hbar=1$.

The time evolution of the system is given by the timedependent Schrödinger equation in atomic units:

$$
i \partial_{t}|\Psi, t\rangle=\hat{H}(t)|\Psi, t\rangle .
$$

We assume that we have solved the FIR-only problem using the initial condition for the time evolution operator:

$$
\hat{U}_{\mathrm{FIR}}(t,-\infty) \rightarrow \exp \left[-i\left(\hat{H}_{0 \mathrm{~A}}-E_{0}\right) t\right] \text { as } t \rightarrow-\infty \text {. }
$$

Therefore in the absence of the perturbation, i.e., $\lambda=0$, the solution of Eq. (A2) is

$$
\left|\Psi_{\mathrm{FIR}}, t\right\rangle \equiv \hat{U}_{\mathrm{FIR}}(t,-\infty)\left|\Psi_{0}\right\rangle,
$$

where $\left|\Psi_{0}\right\rangle$ is the ground state of the Mg atom. Now we make the ansatz

$$
|\Psi, t\rangle=|\Psi, t\rangle^{(0)}+\lambda|\Psi, t\rangle^{(1)}+\lambda^{2}|\Psi, t\rangle^{(2)}+\cdots,
$$

where $|\Psi, t\rangle^{(0)}=\left|\Psi_{\mathrm{FIR}}, t\right\rangle,|\Psi, t\rangle^{(1)}$, and $|\Psi, t\rangle^{(2)}$ are the zero, first- and second-order corrected wave functions. The correction terms are calculated by substituting Eqs. (A5) and (A1) in Eq. (A2), which gives

$$
\begin{aligned}
i \partial_{t}\left[\left|\Psi_{\mathrm{FIR}}, t\right\rangle+\lambda|\Psi, t\rangle^{(1)}+\lambda^{2}|\Psi, t\rangle^{(2)}\right] \\
=\left[\hat{H}_{0}(t)+\lambda \hat{V}(t)\right]\left[\left|\Psi_{\mathrm{FIR}}, t\right\rangle\right. \\
\left.\quad+\lambda|\Psi, t\rangle^{(1)}+\lambda^{2}|\Psi, t\rangle^{(2)}\right]
\end{aligned}
$$

Now comparing the coefficients of the $\lambda$ terms from both the right-hand side (RHS) and the left-hand side (LHS) in
Eq. (A6), we obtain the time evolution of the first-order correction term:

$$
i \partial_{t}|\Psi, t\rangle^{(1)}=\hat{H}_{0}(t)|\Psi, t\rangle^{(1)}+\hat{V}(t)\left|\Psi_{\mathrm{FIR}}, t\right\rangle,
$$

which can be solved as a first-order differential equation with variable coefficients. The solution is

$$
\begin{gathered}
|\Psi, t\rangle^{(1)}=-i \hat{U}_{\mathrm{FIR}}(t,-\infty) \int_{-\infty}^{t} d t^{\prime} \hat{U}_{\mathrm{FIR}}^{\dagger}\left(t^{\prime},-\infty\right) \hat{V}\left(t^{\prime}\right)\left|\Psi_{\mathrm{FIR}}, t^{\prime}\right\rangle, \\
|\Psi, t\rangle^{(1)}=-i \int_{-\infty}^{t} d t^{\prime} \hat{U}_{\mathrm{FIR}}\left(t, t^{\prime}\right) \hat{V}\left(t^{\prime}\right)\left|\Psi_{\mathrm{FIR}}, t^{\prime}\right\rangle,
\end{gathered}
$$

where $\hat{U}_{\mathrm{FIR}}\left(t, t^{\prime}\right)=U_{\mathrm{FIR}}(t,-\infty) \hat{U}_{\mathrm{FIR}}^{\dagger}\left(t^{\prime},-\infty\right)$. Comparing the coefficients of $\lambda^{2}$ terms from both the RHS and the LHS in Eq. (A6), we obtain the time evolution of the second-order correction term:

$$
i \partial_{t}|\Psi, t\rangle^{(2)}=\hat{H}_{0}(t)|\Psi, t\rangle^{(2)}+\hat{V}(t)|\Psi, t\rangle^{(1)} .
$$

It can be solved similarily as the first-order correction term, which gives

$$
|\Psi, t\rangle^{(2)}=-i \int_{-\infty}^{t} d t^{\prime} \hat{U}_{\mathrm{FIR}}\left(t, t^{\prime}\right) \hat{V}\left(t^{\prime}\right)\left|\Psi, t^{\prime}\right\rangle^{(1)} .
$$

Substituting Eq. (A9) into Eq. (A11), we get

$$
\begin{aligned}
|\Psi, t\rangle^{(2)}= & -\int_{-\infty}^{t} d t^{\prime} \int_{-\infty}^{t^{\prime}} d t^{\prime \prime} \hat{U}_{\mathrm{FIR}}\left(t, t^{\prime}\right) \hat{V}\left(t^{\prime}\right) \hat{U}_{\mathrm{FIR}} \\
& \times\left(t^{\prime}, t^{\prime \prime}\right) \hat{V}\left(t^{\prime \prime}\right)\left|\Psi_{\mathrm{FIR}}, t^{\prime \prime}\right\rangle
\end{aligned}
$$

Using $\hat{V}(t)=\hat{V}_{\mathrm{EUV}}(t)+\hat{V}_{\mathrm{A}}$ and Eq. (A4), we obtain

$$
\begin{aligned}
|\Psi, t\rangle^{(2)}= & -\int_{-\infty}^{t} d t^{\prime} \int_{-\infty}^{t^{\prime}} d t^{\prime \prime} \hat{U}_{\mathrm{FIR}}\left(t, t^{\prime}\right)\left[\hat{V}_{\mathrm{EUV}}\left(t^{\prime}\right)\right. \\
& \left.+\hat{V}_{\mathrm{A}}\right] \hat{U}_{\mathrm{FIR}}\left(t^{\prime}, t^{\prime \prime}\right) \\
& \times\left[\hat{V}_{\mathrm{EUV}}\left(t^{\prime \prime}\right)+\hat{V}_{\mathrm{A}}\right] \hat{U}_{\mathrm{FIR}}\left(t^{\prime \prime},-\infty\right)\left|\Psi_{0}\right\rangle .
\end{aligned}
$$

Out of four terms in the above equation, we are interested in the term in which there is first EUV photoionization and then Auger decay. The second-order corrected wave function for the corresponding process is

$$
\begin{aligned}
|\Psi, t\rangle^{(2)}= & -\int_{-\infty}^{t} d t^{\prime} \int_{-\infty}^{t^{\prime}} d t^{\prime \prime} \hat{U}_{\mathrm{FIR}}\left(t, t^{\prime}\right) \hat{V}_{\mathrm{A}} \hat{U}_{\mathrm{FIR}}\left(t^{\prime}, t^{\prime \prime}\right) \\
& \times \hat{V}_{\mathrm{EUV}}\left(t^{\prime \prime}\right) \hat{U}_{\mathrm{FIR}}\left(t^{\prime \prime},-\infty\right)\left|\Psi_{0}\right\rangle .
\end{aligned}
$$

We choose the polarization direction of the EUV pulse along the $z$ axis to obtain $\hat{V}_{\mathrm{EUV}}\left(t^{\prime \prime}\right)=\varepsilon_{\mathrm{EUV}}\left(t^{\prime \prime}\right) \hat{Z}$, where $\varepsilon_{\mathrm{EUV}}$ describes the time evolution of the EUV pulse. We further assume that the FIR field is too weak to perturb the ground state. Hence, $\hat{U}_{\mathrm{FIR}}\left(t^{\prime \prime},-\infty\right)\left|\Psi_{0}\right\rangle=e^{-i\left(\hat{H}_{0 \mathrm{~A}}-E_{0}\right) t^{\prime \prime}}\left|\Psi_{0}\right\rangle=\left|\Psi_{0}\right\rangle$. Substituting both relations into Eq. (A14) gives

$$
\begin{aligned}
|\Psi, t\rangle^{(2)}= & -\int_{-\infty}^{t} d t^{\prime} \int_{-\infty}^{t^{\prime}} d t^{\prime \prime} \hat{U}_{\mathrm{FIR}}\left(t, t^{\prime}\right) \hat{V}_{\mathrm{A}} \hat{U}_{\mathrm{FIR}} \\
& \times\left(t^{\prime}, t^{\prime \prime}\right) \varepsilon_{\mathrm{EUV}}\left(t^{\prime \prime}\right) \hat{Z}\left|\Psi_{0}\right\rangle .
\end{aligned}
$$

We now define the one-hole-one-particle wave functions, $\left|\Psi_{k}^{b}\right\rangle$ and $\left|\Psi_{j}^{a}\right\rangle$, and the two-hole-two-particle wave functions, $\left|\Psi_{k^{\prime} k^{\prime \prime}}^{b^{\prime} b^{\prime \prime}}\right\rangle$ and $\left|\Psi_{j^{\prime} j^{\prime \prime}}^{a^{\prime} a^{\prime \prime}}\right\rangle$, in which the subscript indices, 
e.g., $k$ and $j$, represent the holes and the superscript indices, e.g., $a$ and $b$, represent the particles. These onehole-one-particle and two-hole-two-particle wave functions are defined relative to $\left|\Psi_{0}\right\rangle$, which we assume to be a single Slater determinant, and are eigenstates of the unperturbed atomic Hamiltonian $\hat{H}_{0 A}$, which we assume to be a mean-field Hamiltonian. We insert four completeness relations $\sum_{j}\left|\Psi_{j}^{a}\right\rangle\left\langle\Psi_{j}^{a}\left|, \sum_{b}\right| \Psi_{k}^{b}\right\rangle\left\langle\Psi_{k}^{b}\left|, \sum_{\substack{j^{\prime \prime}>j^{\prime} \\ a^{\prime \prime}}}\right| \Psi_{j^{\prime} j^{\prime \prime}}^{a^{\prime} a^{\prime \prime}}\right\rangle\left\langle\Psi_{j^{\prime} j^{\prime \prime}}^{a^{\prime} a^{\prime \prime}}\right|$, and $\sum_{\substack{k^{\prime \prime}>k^{\prime} \\ b^{\prime \prime}>b^{\prime}}}\left|\Psi_{k^{\prime} k^{\prime \prime}}^{b^{\prime} b^{\prime \prime}}\right\rangle\left\langle\Psi_{k^{\prime} k^{\prime \prime}}^{b^{\prime} b^{\prime \prime}}\right|$ into Eq. (A15) and obtain

$$
\begin{aligned}
|\Psi, t\rangle^{(2)}= & -\sum_{\substack{k^{\prime \prime}>k^{\prime} \\
b^{\prime \prime}>b^{\prime}}} \sum_{\substack{j^{\prime \prime}>j^{\prime} \\
a^{\prime \prime}>a^{\prime}}} \sum_{k} \sum_{j} \int_{-\infty}^{t} d t^{\prime} \int_{-\infty}^{t^{\prime}} d t^{\prime \prime}\left|\Psi_{k^{\prime} k^{\prime \prime}}^{b^{\prime} b^{\prime \prime}}\right\rangle\left\langle\Psi_{k^{\prime} k^{\prime \prime}}^{b^{\prime} b^{\prime \prime}}\left|\hat{U}_{\mathrm{FIR}}\left(t, t^{\prime}\right)\right| \Psi_{j^{\prime} j^{\prime \prime}}^{a^{\prime} a^{\prime \prime}}\right\rangle \\
& \times\left\langle\Psi_{j^{\prime} j^{\prime \prime}}^{a^{\prime} a^{\prime \prime}}\left|\hat{V}_{\mathrm{A}}\right| \Psi_{k}^{b}\right\rangle\left\langle\Psi_{k}^{b}\left|\hat{U}_{\mathrm{FIR}}\left(t^{\prime}, t^{\prime \prime}\right)\right| \Psi_{j}^{a}\right\rangle \varepsilon_{\mathrm{EUV}}\left(t^{\prime \prime}\right)\left\langle\Psi_{j}^{a}|\hat{Z}| \Psi_{0}\right\rangle .
\end{aligned}
$$

As $\hat{Z}$ is a one-body operator we can use the Slater-Condon rules to evaluate $\left\langle\Psi_{j}^{a}|\hat{Z}| \Psi_{0}\right\rangle$, which gives $\left\langle\varphi_{a}|\hat{Z}| \varphi_{j}\right\rangle$, where $\varphi_{a}$ and $\varphi_{j}$ are atomic spin orbitals. We assume that the FIR field does not induce particle-hole excitations. We further assume that the FIR field does not have any effect on the holes but only on the particles. Therefore, $\left\langle\Psi_{k}^{b}\left|\hat{U}_{\mathrm{FIR}}\left(t^{\prime}, t^{\prime \prime}\right)\right| \Psi_{j}^{a}\right\rangle$ reduces to $\delta_{j k} u_{b a}$, where $u_{b a}$ is the transition amplitude of the photoelectron from $a$ to $b$ due to the FIR field. Thus we obtain

$$
\begin{aligned}
|\Psi, t\rangle^{(2)}= & -\sum_{\substack{k^{\prime \prime}>k^{\prime} \\
b^{\prime \prime}>b^{\prime}}} \sum_{\substack{j^{\prime \prime}>j^{\prime} \\
a^{\prime \prime}>a^{\prime}}} \sum_{\substack{b \\
b^{\prime}}} \sum_{j} \int_{-\infty}^{t} d t^{\prime} \int_{-\infty}^{t^{\prime}} d t^{\prime \prime}\left|\Psi_{k^{\prime} k^{\prime \prime}}^{b^{\prime} b^{\prime \prime}}\right\rangle\left\langle\Psi_{k^{\prime} k^{\prime \prime}}^{b^{\prime} b^{\prime \prime}}\left|\hat{U}_{\mathrm{FIR}}\left(t, t^{\prime}\right)\right| \Psi_{j^{\prime} j^{\prime \prime}}^{a^{\prime} a^{\prime \prime}}\right\rangle \\
& \times\left\langle\Psi_{j^{\prime} j^{\prime \prime}}^{a^{\prime} a^{\prime \prime}}\left|\hat{V}_{\mathrm{A}}\right| \Psi_{k}^{b}\right\rangle \delta_{j k} u_{b a}\left(t^{\prime}, t^{\prime \prime}\right) e^{-i I_{j}\left(t^{\prime}-t^{\prime \prime}\right)} \varepsilon_{\mathrm{EUV}}\left(t^{\prime \prime}\right)\left\langle\varphi_{a}|\hat{Z}| \varphi_{j}\right\rangle,
\end{aligned}
$$

where $I_{j}=-\epsilon_{j}$ is the ionization energy to remove a particle from orbital $j$. Utilizing the Kronecker $\delta$ we can remove the summation over $k$ and obtain

$$
\begin{aligned}
|\Psi, t\rangle^{(2)}= & -\sum_{\substack{k^{\prime \prime}>k^{\prime} \\
b^{\prime \prime}>b^{\prime}}} \sum_{\substack{j^{\prime \prime}>j^{\prime} \\
a^{\prime \prime}>a^{\prime}}} \sum_{b} \sum_{j} \int_{-\infty}^{t} d t^{\prime} \int_{-\infty}^{t^{\prime}} d t^{\prime \prime}\left|\Psi_{k^{\prime} k^{\prime \prime}}^{b^{\prime} b^{\prime \prime}}\right\rangle\left\langle\Psi_{k^{\prime} k^{\prime \prime}}^{b^{\prime} b^{\prime \prime}}\left|\hat{U}_{\mathrm{FIR}}\left(t, t^{\prime}\right)\right| \Psi_{j^{\prime} j^{\prime \prime}}^{a^{\prime} a^{\prime \prime}}\right\rangle \\
& \times\left\langle\Psi_{j^{\prime} j^{\prime \prime}}^{a^{\prime} a^{\prime \prime}}\left|\hat{V}_{A}\right| \Psi_{j}^{b}\right\rangle u_{b a}\left(t^{\prime}, t^{\prime \prime}\right) e^{-i I_{j}\left(t^{\prime}-t^{\prime \prime}\right)} \varepsilon_{\mathrm{EUV}}\left(t^{\prime \prime}\right) z_{a j} .
\end{aligned}
$$

The two-body operator $\hat{V}_{\mathrm{A}}$ does not affect the ejected photoelectron which gives a condition on the index $a^{\prime}$, i.e., $a^{\prime}=b$ and the summation of $a^{\prime}$ can be removed to obtain

$$
\begin{aligned}
|\Psi, t\rangle^{(2)}= & -\sum_{\substack{k^{\prime \prime}>k^{\prime} \\
b^{\prime \prime}>b^{\prime}}} \sum_{\substack{j^{\prime \prime}>j^{\prime} \\
a^{\prime \prime}>b}} \sum_{j} \int_{-\infty}^{t} d t^{\prime} \int_{-\infty}^{t^{\prime}} d t^{\prime \prime}\left|\Psi_{k^{\prime} k^{\prime \prime}}^{b^{\prime} b^{\prime \prime}}\right\rangle\left\langle\Psi_{k^{\prime} k^{\prime \prime}}^{b^{\prime} b^{\prime \prime}}\left|\hat{U}_{\mathrm{FIR}}\left(t, t^{\prime}\right)\right| \Psi_{j^{\prime} j^{\prime \prime}}^{b a^{\prime \prime}}\right\rangle \\
& \times\left\langle\Psi_{j^{\prime} j^{\prime \prime}}^{b a^{\prime \prime}}\left|\hat{V}_{\mathrm{A}}\right| \Psi_{j}^{b}\right\rangle u_{b a}\left(t^{\prime}, t^{\prime \prime}\right) e^{-i I_{j}\left(t^{\prime}-t^{\prime \prime}\right)} \varepsilon_{\mathrm{EUV}}\left(t^{\prime \prime}\right) z_{a j} .
\end{aligned}
$$

The term consisting of the two-body operator $\hat{V}_{\mathrm{A}}$ can also be evaluated using Slater-Condon rules, by assuming that the photoelectron, labeled as $b$ in this case, is merely a spectator in the Auger process. Therefore,

$$
\begin{aligned}
|\Psi, t\rangle^{(2)}= & -\sum_{\substack{k^{\prime \prime}>k^{\prime} \\
b^{\prime \prime}>b^{\prime}}} \sum_{\substack{j^{\prime \prime}>j^{\prime} \\
a^{\prime \prime}>b}} \sum_{j} \int_{-\infty}^{t} d t^{\prime} \int_{-\infty}^{t^{\prime}} d t^{\prime \prime}\left|\Psi_{k^{\prime} k^{\prime \prime}}^{b^{\prime} b^{\prime \prime}}\right\rangle\left\langle\Psi_{k^{\prime} k^{\prime \prime}}^{b^{\prime} b^{\prime \prime}}\left|\hat{U}_{\mathrm{FIR}}\left(t, t^{\prime}\right)\right| \Psi_{j^{\prime} j^{\prime \prime}}^{b a^{\prime \prime}}\right\rangle \\
& \times v_{a^{\prime \prime} j j^{\prime} j^{\prime \prime}} u_{b a}\left(t^{\prime}, t^{\prime \prime}\right) e^{-i I_{j}\left(t^{\prime}-t^{\prime \prime}\right)} \varepsilon_{\mathrm{EUV}}\left(t^{\prime \prime}\right) z_{a j} .
\end{aligned}
$$

Here, $v_{a^{\prime \prime} j j^{\prime} j^{\prime \prime}}=\left\langle\varphi_{a^{\prime \prime}} \varphi_{j}\left|\hat{V}_{A}\right| \varphi_{j^{\prime}} \varphi_{j^{\prime \prime}}\right\rangle-\left\langle\varphi_{a^{\prime \prime}} \varphi_{j}\left|\hat{V}_{A}\right| \varphi_{j^{\prime \prime}} \varphi_{j^{\prime}}\right\rangle$, where $\varphi_{a^{\prime \prime}}, \varphi_{j}, \varphi_{j^{\prime}}$ and $\varphi_{j^{\prime \prime}}$ are again the atomic spin orbitals. As before, we assume that the FIR field does not affect the holes, but only the particles, which reduces $\left\langle\Psi_{k^{\prime} k^{\prime \prime}}^{b^{\prime} b^{\prime \prime}}\left|\hat{U}_{\mathrm{FIR}}\left(t, t^{\prime}\right)\right| \Psi_{j^{\prime} j^{\prime \prime}}^{b a^{\prime \prime}}\right\rangle$ to $\delta_{j^{\prime} k^{\prime}} \delta_{j^{\prime \prime} k^{\prime \prime}} u_{b^{\prime} b}\left(t, t^{\prime}\right) u_{b^{\prime \prime} a^{\prime \prime}}\left(t, t^{\prime}\right)$, where $u_{b^{\prime} b}\left(t, t^{\prime}\right)$ and $u_{b^{\prime \prime} a^{\prime \prime}}\left(t, t^{\prime}\right)$ are the transition amplitudes of the photoelectron from $b$ to $b^{\prime}$ and of the Auger electron from $a^{\prime \prime}$ to $b^{\prime \prime}$, respectively:

$$
\begin{aligned}
& |\Psi, t\rangle^{(2)}=-\sum_{\substack{k^{\prime \prime}>k^{\prime} \\
b^{\prime \prime}>b^{\prime}}} \sum_{\substack{j^{\prime \prime}>j^{\prime} \\
a^{\prime \prime}>b}} \sum_{j} \int_{-\infty}^{t} d t^{\prime} \int_{-\infty}^{t^{\prime}} d t^{\prime \prime}\left|\Psi_{k^{\prime} k^{\prime \prime}}^{b^{\prime \prime}}\right\rangle \delta_{j^{\prime} k^{\prime}} \delta_{j^{\prime \prime} k^{\prime \prime}} u_{b^{\prime} b}\left(t, t^{\prime}\right) u_{b^{\prime \prime} a^{\prime \prime}}\left(t, t^{\prime}\right) \\
& \times e^{-i I_{j^{\prime} j^{\prime \prime}}\left(t-t^{\prime}\right)} v_{a^{\prime \prime} j j^{\prime} j^{\prime \prime}} u_{b a}\left(t^{\prime}, t^{\prime \prime}\right) e^{-i I_{j}\left(t^{\prime}-t^{\prime \prime}\right)} \varepsilon_{\mathrm{EUV}}\left(t^{\prime \prime}\right) z_{a j} .
\end{aligned}
$$


Here, $I_{j^{\prime} j^{\prime \prime}}$ is the eigenenergy of the double-hole state with holes in the orbitals $j$ and $j^{\prime}$. Resolving the Kronecker $\delta$ 's further removes the summation over $k^{\prime}$ and $k^{\prime \prime}$ :

$$
\begin{aligned}
|\Psi, t\rangle^{(2)}= & \left.-\sum_{b^{\prime \prime}>b^{\prime} j^{\prime \prime}>j^{\prime}} \sum_{\substack{j \\
a^{\prime \prime}>b}} \int_{-\infty}^{t} d t^{\prime} \int_{-\infty}^{t^{\prime}} d t^{\prime \prime} \mid \Psi_{j^{\prime} j^{\prime \prime}}^{b^{\prime} b^{\prime \prime}}\right) u_{b^{\prime} b}\left(t, t^{\prime}\right) u_{b^{\prime \prime} a^{\prime \prime}}\left(t, t^{\prime}\right) \\
& \times e^{-i I_{j^{\prime} j^{\prime \prime}}\left(t-t^{\prime}\right)} v_{a^{\prime \prime} j j^{\prime} j^{\prime \prime}} u_{b a}\left(t^{\prime}, t^{\prime \prime}\right) e^{-i I_{j}\left(t^{\prime}-t^{\prime \prime}\right)} \varepsilon_{\operatorname{EUV}}\left(t^{\prime \prime}\right) z_{a j} .
\end{aligned}
$$

The observable connected to the Auger-electron spectrum is defined as a sum over projectors onto the Auger-electron state $\alpha$ :

$$
\hat{P}_{\alpha}=\sum_{\substack{\gamma>\beta \\ \Pi(\Pi<\alpha)}}\left|\Psi_{\beta \gamma}^{\Pi \alpha}\right\rangle\left\langle\Psi_{\beta \gamma}^{\Pi \alpha}\right|,
$$

where $\left|\Psi_{\beta \gamma}^{\Pi \alpha}\right\rangle$ is a two-hole-two-particle eigenstate of the unperturbed atomic Hamiltonian $\hat{H}_{0 A}$. Now we take the expectation value with the second-order corrected state vector:

$$
\begin{aligned}
P_{\alpha}= & \sum_{\substack{\gamma>\beta \\
\Pi(\Pi<\alpha)}}\left|\left\langle\Psi_{\beta \gamma}^{\Pi \alpha} \mid \Psi, t^{(2)}\right\rangle\right|^{2}=\sum_{\substack{\gamma>\beta \\
\Pi(\Pi<\alpha)}} \mid \sum_{\substack{b^{\prime \prime}>b^{\prime} \\
j^{\prime \prime}>j^{\prime} \\
a^{\prime \prime}>b}} \sum_{\substack{j \\
a}} \int_{-\infty}^{t} d t^{\prime} \int_{-\infty}^{t^{\prime}} d t^{\prime \prime}\left\langle\Psi_{\beta \gamma}^{\Pi \alpha} \mid \Psi_{j^{\prime} j^{\prime \prime}}^{b^{\prime} b^{\prime \prime}}\right\rangle \\
& \times\left. u_{b^{\prime} b}\left(t, t^{\prime}\right) u_{b^{\prime \prime} a^{\prime \prime}}\left(t, t^{\prime}\right) e^{-i I_{j^{\prime} j^{\prime \prime}}\left(t-t^{\prime}\right)} v_{a^{\prime \prime} j j^{\prime} j^{\prime \prime}} u_{b a}\left(t^{\prime}, t^{\prime \prime}\right) e^{-i I_{j}\left(t^{\prime}-t^{\prime \prime}\right)} \varepsilon_{\mathrm{EUV}}\left(t^{\prime \prime}\right) z_{a j}\right|^{2}
\end{aligned}
$$

Since, by assumption, both $\left|\Psi_{\beta \gamma}^{\Pi \alpha}\right\rangle$ and $\left|\Psi_{j^{\prime} j^{\prime \prime}}^{b^{\prime \prime}}\right\rangle$ are eigenstates of the unperturbed atomic Hamiltonian $\hat{H}_{0 A}$, we obtain

$$
\begin{aligned}
P_{\alpha}= & \sum_{\substack{\gamma>\beta \\
\Pi(\Pi<\alpha)}} \mid \sum_{\substack{b^{\prime \prime}>b^{\prime} j^{\prime \prime}>j^{\prime} \\
a^{\prime \prime}>b}} \sum_{\substack{j \\
a}} \int_{-\infty}^{t} d t^{\prime} \int_{-\infty}^{t^{\prime}} d t^{\prime \prime} \delta_{\beta j^{\prime}} \delta_{\gamma j^{\prime \prime}} \delta_{\Pi b^{\prime}} \delta_{\alpha b^{\prime \prime}} u_{b^{\prime} b}\left(t, t^{\prime}\right) u_{b^{\prime \prime} a^{\prime \prime}}\left(t, t^{\prime}\right) \\
& \times\left. e^{-i I_{j^{\prime} \prime^{\prime \prime}}\left(t-t^{\prime}\right)} v_{a^{\prime \prime} j j^{\prime} j^{\prime \prime}} u_{b a}\left(t^{\prime}, t^{\prime \prime}\right) e^{-i I_{j}\left(t^{\prime}-t^{\prime \prime}\right)} \varepsilon_{\mathrm{EUV}}\left(t^{\prime \prime}\right) z_{a j}\right|^{2}
\end{aligned}
$$

Thus,

$$
P_{\alpha}=\sum_{\substack{\gamma>\beta \\ \Pi(\Pi<\alpha)}}\left|\sum_{a^{\prime \prime}>b} \sum_{j} \int_{-\infty}^{t} d t^{\prime} \int_{-\infty}^{t^{\prime}} d t^{\prime \prime} u_{\Pi b}\left(t, t^{\prime}\right) u_{\alpha a^{\prime \prime}}\left(t, t^{\prime}\right) e^{-i I_{\beta \gamma}\left(t-t^{\prime}\right)} v_{a^{\prime \prime} j \beta \gamma} u_{b a}\left(t^{\prime}, t^{\prime \prime}\right) e^{-i I_{j}\left(t^{\prime}-t^{\prime \prime}\right)} \varepsilon_{\mathrm{EUV}}\left(t^{\prime \prime}\right) z_{a j}\right|^{2}
$$

We use $\sum_{b} u_{\Pi b}\left(t, t^{\prime}\right) u_{b a}\left(t^{\prime}, t^{\prime \prime}\right)=u_{\Pi a}\left(t, t^{\prime \prime}\right)$ in the above equation, which gives

$$
\begin{aligned}
& P_{\alpha}=\sum_{\substack{\gamma>\beta \\
\Pi(\Pi<\alpha)}} \mid \sum_{a^{\prime \prime}} \sum_{\substack{j \\
a}} \int_{-\infty}^{t} d t^{\prime} \int_{-\infty}^{t^{\prime}} d t^{\prime \prime} u_{\alpha a^{\prime \prime}}\left(t, t^{\prime}\right) e^{-i I_{\beta \gamma}\left(t-t^{\prime}\right)} \\
& \times\left. v_{a^{\prime \prime} j \beta \gamma} u_{\Pi a}\left(t, t^{\prime \prime}\right) e^{-i I_{j}\left(t^{\prime}-t^{\prime \prime}\right)} \varepsilon_{\mathrm{EUV}}\left(t^{\prime \prime}\right) z_{a j}\right|^{2} \\
& P_{\alpha}=\sum_{\substack{\gamma>\beta \\
\Pi(\Pi<\alpha)}} \mid \sum_{\substack{a^{\prime \prime} \\
j}} \sum_{\substack{j \\
a}}^{t} d t^{\prime} \int_{-\infty}^{t^{\prime}} d t^{\prime \prime} u_{\alpha a^{\prime \prime}}\left(t, t^{\prime}\right) e^{-i I_{\beta \gamma}\left(t-t^{\prime}\right)} \\
& \times\left. v_{a^{\prime \prime} j \beta \gamma} u_{\Pi a}\left(t, t^{\prime \prime}\right) e^{-i\left(I_{j}-i \frac{\Gamma_{j}}{2}\right)\left(t^{\prime}-t^{\prime \prime}\right)} \varepsilon_{\mathrm{EUV}}\left(t^{\prime \prime}\right) z_{a j}\right|^{2}
\end{aligned}
$$

To account for the exponential decay of the hole $j$, we rewrite $I_{j}$ as $I_{j}-i \frac{\Gamma_{j}}{2}$, where $\Gamma_{j}$ is the decay rate. Hence, 
In Eq. (A28), the terms $u_{\Pi a}\left(t, t^{\prime \prime}\right)$ and $u_{\alpha a^{\prime \prime}}\left(t, t^{\prime}\right)$ represent the FIR dressed photoelectron $(\Pi)$ and the Auger electron $(\alpha)$ after EUV photoionization and Auger decay, respectively. Here we emphasize the assumption that the holes are not affected by the FIR field. This is a generalized expression for the signal one gets within our model. The one-body term $z_{a j}$ and the two-body term $v_{a^{\prime \prime} j \beta \gamma}$ represent the photoinonization and the Auger decay, respectively. The FIR field dressings of photoelectrons and Auger electrons are given by the matrix elements $u_{\Pi a}\left(t, t^{\prime \prime}\right)$ and $u_{\alpha a^{\prime \prime}}\left(t, t^{\prime}\right)$, respectively.

\section{APPENDIX B: APPROXIMATIONS}

To simplify Eq. (A28) and allow for a comparison with the experimental spectrum we first assume the EUV pulse to be a $\delta$ pulse in the form $\varepsilon_{\mathrm{EUV}}(t)=\varepsilon_{0} \delta\left(t-t_{\mathrm{EUV}}\right)$, where $\varepsilon_{0}$ is the strength of the pulse. By using this $\delta$-pulse approximation in Eq. (A28), we get the following for $t>t_{\mathrm{EUV}}$ :

$$
\begin{aligned}
P_{\alpha}= & \sum_{\substack{\gamma>\beta \\
\Pi(\Pi<\alpha)}} \mid \sum_{a^{\prime \prime}} \sum_{j} \int_{t_{\mathrm{EUV}}}^{t} d t^{\prime} u_{\alpha a^{\prime \prime}}\left(t, t^{\prime}\right) e^{-i I_{\beta \gamma}\left(t-t^{\prime}\right)} \\
& \times\left. v_{a^{\prime \prime} j \beta \gamma} u_{\Pi a}\left(t, t_{\mathrm{EUV}}\right) e^{-i\left(I_{j}-i \frac{\Gamma_{j}}{2}\right)\left(t^{\prime}-t_{\mathrm{EUV}}\right)} \varepsilon_{0} z_{a j}\right|^{2} .
\end{aligned}
$$

Second, we look at the form of the equation in the absence of the FIR field. The transition amplitudes are then

$$
\begin{gathered}
u_{\alpha a^{\prime \prime}}\left(t, t^{\prime}\right)=e^{-i \varepsilon_{\alpha}\left(t-t^{\prime}\right)} \delta_{\alpha a^{\prime \prime}}, \\
u_{\Pi a}\left(t, t_{\mathrm{EUV}}\right)=e^{-i \varepsilon_{\Pi}\left(t-t_{\mathrm{EUV}}\right)} \delta_{\Pi a} .
\end{gathered}
$$

Using these, we get the following for $t>t_{\mathrm{EUV}}$ :

$$
\begin{aligned}
P_{\alpha}= & \sum_{\substack{\gamma>\beta \\
\Pi(\Pi<\alpha)}} \mid \sum_{j} \int_{t_{\mathrm{EUV}}}^{t} d t^{\prime} e^{-i \varepsilon_{\alpha}\left(t-t^{\prime}\right)} e^{-i I_{\beta \gamma}\left(t-t^{\prime}\right)} \\
\times & \left.v_{\alpha j \beta \gamma} e^{-i \varepsilon_{\Pi}\left(t-t_{\mathrm{EUV}}\right)} e^{-i\left(I_{j}-i \frac{\Gamma_{j}}{2}\right)\left(t^{\prime}-t_{\mathrm{EUV}}\right)} \varepsilon_{0} z_{\Pi j}\right|^{2} \\
= & \sum_{\substack{\gamma>\beta \\
(\Pi<\alpha)}} \mid \sum_{j} \int_{t_{\mathrm{EUV}}}^{t} d t^{\prime} e^{i \varepsilon_{\alpha} t^{\prime}} e^{i I_{\beta \gamma} t^{\prime}} \\
& \times\left. v_{\alpha j \beta \gamma} e^{i \varepsilon_{\Pi} t_{\mathrm{EUV}}} e^{-i\left(I_{j}-i \frac{\Gamma_{j}}{2}\right)\left(t^{\prime}-t_{\mathrm{EUV}}\right)} \varepsilon_{0} z_{\Pi j}\right|^{2},
\end{aligned}
$$

where we exploited that $\left|e^{-i\left(I_{\beta \gamma}+\varepsilon_{\alpha}+\varepsilon_{\Pi}\right) t}\right|^{2}=1$. By carrying out the integral and expanding the modulus, the resulting expression for the Auger-electron spectrum, in the limit $t \rightarrow \infty$, will be in the form of Lorentzians, peaked at the energies $I_{j}-I_{\beta \gamma}$.

To approximately take the FIR field into account, we employ Eq. (B1) and make the following ansatz for the transition amplitudes:

$$
\begin{gathered}
u_{\alpha a^{\prime \prime}}\left(t, t^{\prime}\right)=e^{-i \epsilon_{\alpha}\left(t-t^{\prime}\right)} e^{-i b t^{\prime 2}} g_{\alpha a^{\prime \prime}}, \\
u_{\Pi a}\left(t, t_{\mathrm{EUV}}\right)=e^{-i \epsilon_{\Pi}\left(t-t_{\mathrm{EUV}}\right)} g_{\Pi a} .
\end{gathered}
$$

This ansatz is motivated by the following two observations. First, we are interested in the limit of large $t$, i.e., much larger than the duration of the FIR pulse. At large times $t$, both the photoelectron and the Auger electron propagate freely. Second, $t^{\prime}$ is constrained by the exponential decay to an interval that is short in comparison to the FIR cycle and, thus, also in comparison to the FIR pulse duration.

In this way, we take into consideration a leading-order FIR-induced electronic chirp on the timescale governed by the Auger decay. Moreover, by replacing the previous Kronecker $\delta$ 's in Eqs. (B2) and (B3), with $g_{\alpha a^{\prime \prime}}$ and $g_{\Pi a}$, we take into consideration that the FIR field can change the state of both the photoelectron and the Auger electron.

We insert the expressions for the electronic transition amplitudes given in Eqs. (B6) and (B7) into Eq. (B1):

$$
\begin{aligned}
P_{\alpha}= & \sum_{\substack{\gamma>\beta \\
\Pi(\Pi<\alpha)}} \mid \sum_{j} \int_{t_{\mathrm{EUV}}}^{t} d t^{\prime} e^{-i I_{\beta \gamma}\left(t-t^{\prime}\right)} e^{-i \epsilon_{\alpha}\left(t-t^{\prime}\right)} e^{-i b t^{\prime 2}} \\
& \times e^{-i\left(I_{j}-i \frac{\Gamma_{j}}{2}\right)\left(t^{\prime}-t_{\mathrm{EUV}}\right)} e^{-i \epsilon_{\Pi}\left(t-t_{\mathrm{EUV}}\right)} \\
& \times\left.\underbrace{\sum_{a a^{\prime \prime}} v_{a^{\prime \prime} j \beta \gamma} g_{\alpha a^{\prime \prime}} g_{\Pi a} \varepsilon_{0} z_{a j}}_{A_{j \alpha \beta \gamma}}\right|^{2} .
\end{aligned}
$$

By adding and removing suitable complex phase factors of modulus one, performing the substitution $t^{\prime}-t_{\mathrm{EUV}} \rightarrow t^{\prime}$, and taking the limit $t \rightarrow \infty$, Eq. (B8) goes over into

$$
\begin{aligned}
P_{\alpha}= & \sum_{\substack{\gamma>\beta \\
\Pi(\Pi<\alpha)}} \mid \sum_{j} A_{j \alpha \beta \gamma \Pi} \int_{0}^{\infty} d t^{\prime} e^{-i\left(I_{j}-i \Gamma_{j} / 2-I_{\beta \gamma}-\varepsilon_{\alpha}\right) t^{\prime}} \\
& \times\left. e^{-i b\left(t^{\prime}+t_{\mathrm{EUV}}\right)^{2}}\right|^{2} \\
= & \sum_{\substack{\gamma>\beta \\
\Pi(\Pi<\alpha)}}\left|\int_{-\infty}^{\infty} d t^{\prime} \Psi_{\alpha \beta \gamma \Pi}\left(t^{\prime} ; t_{\mathrm{EUV}}\right) e^{i \varepsilon_{\alpha} t^{\prime}}\right|^{2}
\end{aligned}
$$

where we have rewritten the Auger spectrum in the presence of the FIR field in terms of the Fourier transform of transition amplitudes:

$$
\begin{aligned}
& \Psi_{\alpha \beta \gamma \Pi}\left(t^{\prime} ; t_{\mathrm{EUV}}\right) \\
& \quad= \begin{cases}\sum_{j} A_{j \alpha \beta \gamma \Pi} e^{-i\left(I_{j}-i \Gamma_{j} / 2-I_{\beta \gamma}\right) t^{\prime}} e^{-i b\left(t^{\prime}+t_{\mathrm{EUV}}\right)^{2}}, & t^{\prime} \geqslant 0, \\
0, & t^{\prime}<0 .\end{cases}
\end{aligned}
$$


The coefficients $A_{j \alpha \beta \gamma \Pi}$, defined in Eq. (B8), are complex numbers carrying phase information from photoionization (argument of $z_{a j}$ ) and Auger decay (argument of $v_{a^{\prime \prime} j \beta \gamma}$ ), and from the FIR dressing of the photoelectron (argument of $g_{\Pi a}$ ) and the Auger electron (argument of $g_{\alpha a^{\prime \prime}}$ ).

Equation (B9) is consistent with the expectation that, if $b=0$, the time $t_{\mathrm{EUV}}$ at which the pump $\delta$ pulse is assumed to be centered has no consequence for the Auger spectrum. Only if $b \neq 0$ (i.e., in the presence of the FIR field) does the transition amplitude in Eq. (B10) and, therefore, the Auger spectrum depend explicitly on $t_{\mathrm{EUV}}$. This, in turn, implies that the Auger spectrum must depend on the distribution of $t_{\mathrm{EUV}}$ values, as defined by the envelope of the actual (non- $\delta$ ) EUV field.
[1] D. Attwood, Soft X-Rays and Extreme Ultraviolet Radiation: Principles and Applications (Cambridge University, Cambridge, England, 1999).

[2] J. Als-Nielsen and D. McMorrow, Elements of Modern X-Ray Physics (Wiley \& Sons, New York, 2011).

[3] L. Young, K. Ueda, M. Gühr, P. H. Bucksbaum, M. Simon, S. Mukamel, N. Rohringer, K. C. Prince, C. Masciovecchio, M. Meyer et al., J. Phys. B: At., Mol. Opt. Phys. 51, 032003 (2018).

[4] T. Glover, D. Fritz, M. Cammarata, T. Allison, S. Coh, J. Feldkamp, H. Lemke, D. Zhu, Y. Feng, R. Coffee et al., Nature (London) 488, 603 (2012).

[5] C. Buth, R. Santra, and L. Young, Phys. Rev. Lett. 98, 253001 (2007).

[6] T. Glover, M. Hertlein, S. Southworth, T. Allison, J. van Tilborg, E. Kanter, B. Krässig, H. Varma, B. Rude, R. Santra et al., Nat. Phys. 6, 69 (2010).

[7] E. R. Peterson, C. Buth, D. A. Arms, R. W. Dunford, E. P. Kanter, B. Krässig, E. C. Landahl, S. T. Pratt, R. Santra, S. H. Southworth et al., Appl. Phys. Lett. 92, 094106 (2008).

[8] C. Buth and R. Santra, J. Chem. Phys. 129, 134312 (2008).

[9] C. Ott, A. Kaldun, P. Raith, K. Meyer, M. Laux, J. Evers, C. H. Keitel, C. H. Greene, and T. Pfeifer, Science 340, 716 (2013).

[10] A. Kaldun, A. Blättermann, V. Stooß, S. Donsa, H. Wei, R. Pazourek, S. Nagele, C. Ott, C. Lin, J. Burgdörfer et al., Science 354, 738 (2016).

[11] I. Grguraš, A. R. Maier, C. Behrens, T. Mazza, T. Kelly, P. Radcliffe, S. Düsterer, A. Kazansky, N. Kabachnik, T. Tschentscher et al., Nat. Photonics 6, 852 (2012).

[12] W. Helml, A. Maier, W. Schweinberger, I. Grguraš, P. Radcliffe, G. Doumy, C. Roedig, J. Gagnon, M. Messerschmidt, S. Schorb et al., Nat. Photonics 8, 950 (2014).

[13] T. Mazza, M. Ilchen, A. J. Rafipoor, C. Callegari, P. Finetti, O. Plekan, K. C. Prince, R. Richter, M. Danailov, A. Demidovich et al., Nat. Commun. 5, 3648 (2014).

[14] T. E. Glover, R. W. Schoenlein, A. H. Chin, and C. V. Shank, Phys. Rev. Lett. 76, 2468 (1996).

[15] P. M. Paul, E. S. Toma, P. Breger, G. Mullot, F. Augé, P. Balcou, H. G. Muller, and P. Agostini, Science 292, 1689 (2001).

[16] A. Baltuška, T. Udem, M. Uiberacker, M. Hentschel, E. Goulielmakis, C. Gohle, R. Holzwarth, V. Yakovlev, A. Scrinzi, T. W. Hänsch et al., Nature (London) 421, 611 (2003).

[17] M. Drescher, M. Hentschel, R. Kienberger, M. Uiberacker, V. Yakovlev, A. Scrinzi, T. Westerwalbesloh, U. Kleineberg, U. Heinzmann, and F. Krausz, Nature (London) 419, 803 (2002).

[18] L. Miaja-Avila, C. Lei, M. Aeschlimann, J. L. Gland, M. M. Murnane, H. C. Kapteyn, and G. Saathoff, Phys. Rev. Lett. 97, 113604 (2006).
[19] K. Hütten, M. Mittermair, S. O. Stock, R. Beerwerth, V. Shirvanyan, J. Riemensberger, A. Duensing, R. Heider, M. S. Wagner, A. Guggenmos et al., Nat. Commun. 9, 719 (2018).

[20] J. M. Schins, P. Breger, P. Agostini, R. C. Constantinescu, H. G. Muller, G. Grillon, A. Antonetti, and A. Mysyrowicz, Phys. Rev. Lett. 73, 2180 (1994).

[21] M. Meyer, P. Radcliffe, T. Tschentscher, J. T. Costello, A. L. Cavalieri, I. Grguras, A. R. Maier, R. Kienberger, J. Bozek, C. Bostedt et al., Phys. Rev. Lett. 108, 063007 (2012).

[22] P. Ranitovic, X. M. Tong, C. W. Hogle, X. Zhou, Y. Liu, N. Toshima, M. M. Murnane, and H. C. Kapteyn, Phys. Rev. Lett. 106, 053002 (2011).

[23] B. Cooper and V. Averbukh, Phys. Rev. Lett. 111, 083004 (2013).

[24] D. Iablonskyi, K. Ueda, K. L. Ishikawa, A. S. Kheifets, P. Carpeggiani, M. Reduzzi, H. Ahmadi, A. Comby, G. Sansone, T. Csizmadia et al., Phys. Rev. Lett. 119, 073203 (2017).

[25] U. Frühling, M. Wieland, M. Gensch, T. Gebert, B. Schütte, M. Krikunova, R. Kalms, F. Budzyn, O. Grimm, J. Rossbach et al., Nat. Photonics 3, 523 (2009).

[26] B. Schütte, U. Frühling, M. Wieland, A. Azima, and M. Drescher, Opt. Express 19, 18833 (2011).

[27] B. Schütte, S. Bauch, U. Frühling, M. Wieland, M. Gensch, E. Plönjes, T. Gaumnitz, A. Azima, M. Bonitz, and M. Drescher, Phys. Rev. Lett. 108, 253003 (2012).

[28] O. Schwarzkopf and V. Schmidt, J. Phys. B: At., Mol. Opt. Phys. 29, 3023 (1996).

[29] E. Kukk, H. Aksela, A. Kivimäki, J. Jauhiainen, E. Nõmmiste, and S. Aksela, Phys. Rev. A 56, 1481 (1997).

[30] R. Camilloni, M. Žitnik, C. Comicioli, K. C. Prince, M. Zacchigna, C. Crotti, C. Ottaviani, C. Quaresima, P. Perfetti, and G. Stefani, Phys. Rev. Lett. 77, 2646 (1996).

[31] A. Niehaus, J. Phys. B: At. Mol. Phys. 10, 1845 (1977).

[32] S. Rioual, B. Rouvellou, L. Avaldi, G. Battera, R. Camilloni, G. Stefani, and G. Turri, Phys. Rev. Lett. 86, 1470 (2001).

[33] M. Neeb, J.-E. Rubensson, M. Biermann, and W. Eberhardt, J. Electron Spectrosc. Relat. Phenom. 67, 261 (1994).

[34] R. Feifel, T. Tanaka, M. Hoshino, H. Tanaka, Y. Tamenori, V. Carravetta, and K. Ueda, Phys. Rev. A 74, 062717 (2006).

[35] C. Vozzi, M. Nisoli, G. Sansone, S. Stagira, and S. De Silvestri, Appl. Phys. B 80, 285 (2005).

[36] J. Hebling, G. Almasi, I. Z. Kozma, and J. Kuhl, Opt. Express 10, 1161 (2002).

[37] B. Breuckmann, V. Schmidt, and W. Schmitz, J. Phys. B: At., Mol. Opt. Phys. 9, 3037 (1976).

[38] L. Forrest, G. James, K. Ross, and V. Pejcev, J. Phys. B: At., Mol. Opt. Phys. 16, 4671 (1983). 